\title{
Performance Evaluation of an Enhanced Distributed Channel Access Protocol under Heterogeneous Traffic
}

\author{
Mamun I. Abu-Tair and Geyong Min \\ Department of Computing, School of Informatics, University of Bradford, Bradford, BD7 1DP, U.K. \\ \{m.i.a.abu-tair, g.min\}@brad.ac.uk
}

\begin{abstract}
Recently there have been considerable interests focusing on the performance evaluation of IEEE 802.11e Medium Access Control (MAC) protocols, which were proposed for supporting Quality of Services (QoS) in Wireless Local Area Networks (WLANs). Different from most existing work, this study has conducted comprehensive performance evaluation and analysis of the IEEE 802.11e Enhanced Distributed Channel Access (EDCA) protocol in the presence of heterogeneous network traffic including non-bursty Poisson, bursty $\mathrm{ON} / \mathrm{OFF}$, and self-similar traffic generated by wireless multimedia applications. The performance results on throughput, access delay and medium utilization have demonstrated that the protocol is able to achieve satisfying QoS differentiation for heterogeneous multimedia traffic. On the other hand the results have showed that IEEE 802.11e EDCA suffering from the low medium utilization due to the overhead generated by transmission collisions and back-off processes.
\end{abstract}

\section{Introduction}

The Wireless Local Area Network (WLAN) is a flexible data communication system that can either replace or extend a wired LAN to provide location independent network access between computation and communication devices using waves rather than a cable infrastructure. WLAN is becoming more widely recognized as a generalpurpose connectivity alternative for a broad range of business organizations owing to its simplicity, scalability, relative ease of integrating wireless access and ability for wireless stations to roam throughout the business organizations with remaining connected to other existing network resources such as servers, printers, and Internet connections. Many practical WLANs have widely employed the Institute of Electrical and Electronics Engineers (IEEE) 802.11 standards ratified in 1997 that can operate at data rates up to $2 \mathrm{Mbps}$ in the $2.4-\mathrm{GHz}$ Industrial, Scientific and Medical (ISM) band. But the most general business requirements cannot be well supported by the slow data rate of the original IEEE 802.11 standard. Recognizing the critical need to support higher data-transmission rates, the IEEE ratified both $802.11 \mathrm{a}$ and $802.11 \mathrm{~b}$ standards with the rates up to 54 and $11 \mathrm{Mbps}$ in the 5 and 2.4-GHz ISM band, respectively [8, 17]. Moreover, both standards specify the specification of the Medium Access Control (MAC) protocol, which is responsible for control access to the transmission medium. The most important purpose of this protocol is to enable the capacity of transmission media to be utilized in an efficient manner by wireless network devices.

The IEEE 802.11 MAC protocol offers two different methods to support share access to wireless channels; a Distributed Coordination Function (DCF) and an optional Point Coordination Function (PCF) [8]. At the present time, the DCF is the dominant MAC mechanism implemented in the IEEE 802.11-compliant products. The DCF is based on the Carrier Sense Multiple Accesses (CSMA) mechanism, which is a contention-based protocol making certain that the stations first sense the medium before data-transmission. Moreover the DCF applies a collision avoidance (CA) mechanism which can reduce the probability of collisions using an additional random binary exponential time called back-off time. The main objective of CSMA/CA is to avoid stations transmitting at the same time, which can lead to collisions and corresponding retransmissions $[3,8,9,17]$. In addition to the common CSMA/CA techniques, the DCF further reduces the possibility of collisions and improves data delivery reliability by adding acknowledgement frames and optional channel reservation frames (i.e., Request-To-Send and Clear-To-Send) to the exchange sequences of its data frames. Different from DCF, the optional coordination function PCF is a centralized scheme designed for infrastructure networks that have a point coordinator operating at the Access Point (AP) to poll and select the next wireless station for data-transmission [8]. 
With the increasing demand of WLANs, the support of differentiated QoS has become a critical issue on the success of IEEE 802.11 MAC protocols for the future wireless communications. It is important to develop new medium access schemes that can support real-time multimedia applications with differentiated QoS requirements over IEEE 802.11 WLANs. As a result, the IEEE 802.11 working group has very recently standardized an extended version (IEEE 802.11e) that defines two mechanisms for the support of QoS differentiation: Enhanced Distributed Channel Access (EDCA) and Hybrid Coordination Function (HCF) Controlled Channel Access (HCCA) [18]. EDCA delivers traffic based on differentiated Traffic Categories (TCs), which can be achieved by varying the amount of time for which a station senses the channel to be idle before backoff, or the length of the contention window to be used for the back-off, or the duration that a station may transmit after acquiring the channel [18]. The stations with lowerpriority traffic must wait longer than those with highpriority traffic before accessing the medium [18].

Performance analysis of IEEE $802.11 \mathrm{e}$ has attracted lots of research efforts $[6,9,10,11,15]$ over the past years. For instance, Mangold et al. [11] have evaluated the performance of IEEE 802.11e in the single and overlapping Access Point (AP) environments using traffic models with negative-exponentially distributed interarrival times. This study has focused on the analysis of the effectiveness and limitations of IEEE 802.11e in such environments. Lindgren et al. $[9,10]$ have investigated and compared different MAC mechanisms to support QoS in WLAN including PCF, Distributed Fair Scheduling (DFS), Blackburst and EDCA. Their results have showed that the best performance is achieved by Blackburst and have demonstrated that PCF and EDCA are able to support good service differentiation. Grilo and Munes [6] have conducted the performance evaluation of IEEE 802.11e under different types of traffic (such as VoIP, video) with the limitation that each station generates only one single class of traffic. Truong and Vannuccini [15] have evaluated the effectiveness of various IEEE 802.11e QoS mechanisms for supporting voice, video and data applications separately in individual scenarios.

Recent research on network traffic measurement has indicated that the compressed Variable-Bit-Rate (VBR) video traffic exhibits self-similar properties (i.e., traffic burstiness and correlations appearing over many time scales) $[2,4]$. Moreover, such self-similar traffic behaviors have been demonstrated to appear in WLANs [14]. However, existing performance studies on IEEE 802.11 EDCA have not considered traffic self-similarity that has great impacts on the performance of MAC protocols and communication networks. Motivated by the need of different models to capture the heterogeneous characteristics of real-world network traffic, this study uses non-bursty Poisson, bursty ON/OFF, and self-similar processes to model traffic generated by various wireless applications in WLANs with the aim of comprehensively investigating and evaluating the performance of EDCA. Voice traffic is modeled by a two-state ON/OFF process emulating talkspurts followed by silence periods. VBR video traffic is captured by self-similar process. Moreover, Background traffic is modeled by Poisson model.

The rest of the paper is organized as follows: Section 2 introduces IEEE 802.11 MAC mechanisms and its extended version, 802.11e EDCA, for QoS support. This section then reviews the background of traffic models. Section 3 describes the simulation scenarios and the setting of simulation parameters. Section 4 presents and analyses the performance results obtained from simulation experiments. Finally, Section 5 concludes this study.

\section{Background}

\subsection{Distributed Coordination Function (DCF)}

The contention-based DCF is the basic access mechanism of IEEE 802.11. Like most of the contentionbased MAC protocols, DCF relies on a Carrier Sense Multiple collision Access with Collision Avoidance (CSMA/CA) algorithm to access the shared medium. A station having packets ready for transmission senses whether or not the medium is busy. If it has been idle for longer than the minimum duration called DCF Interference Space (DIFS) the station can start transmission immediately. Otherwise a back-off time is chosen randomly from the interval $[0, c w]$, where $c w$ represents the contention window [17]. The stations start down-counting its back-off counter by one only if the medium has been detected idle for at least a DIFS. If the medium gets busy due to other transmissions, the back-off counter pauses down-counting and resumes when the medium has been sensed idle for DIFS again $[8,17]$. Transmission may proceed when back-off counter has reached zero. Upon detection of a collision, i.e., when the back-off counter of two or more stations reach zero at the same time, the contention window is doubled according to $c w_{i}=2^{k+i-1}-1$ where $i$ is number of attempts to transmit the frame and $k$ is a constant defining the minimum contention window $c w_{\min }=2^{k}-1$ [10]. When the destination station receives frame successfully, it sends an acknowledgment (ACK) frame back to the source station after a Short Inter frame Space (SIFS) duration. Additionally, to alleviate the hidden station problem, DCF uses optional Request-to-Send/Clear-to-Send (RTS/CTS) frames before packet transmission [17]. This process has been illustrated in Figure 1. 


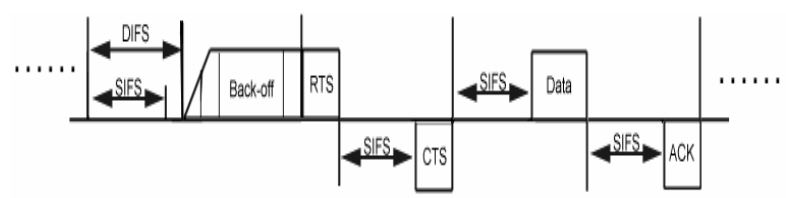

Figure 1: RTS/CTS of IEEE 802.11 DCF

\subsection{Enhanced Distributed Channel Access (EDCA)}

The IEEE 802.11 DCF has no functionality to support QoS requirements of multimedia applications due to its contention-based mechanism. To overcome this drawback and enhance the traditional DCF, EDCA has been proposed to provide differentiated and distributed channel accesses for packets with 8 different Traffic Categories (TCs). As illustrated in Figure 2, individual TC contends to access the medium and is differentiated by different access category Inter-frame Space (AIFS) values, Transmission Opportunity (TXOP) limits and contention windows $\left(c w_{\min }, c w_{\max }\right)[11,12]$. Choosing a smaller contention window for a station will cause the station to gain priority over others with a larger contention window. Each TC has its own transmission queue, as shown in Figure 3.

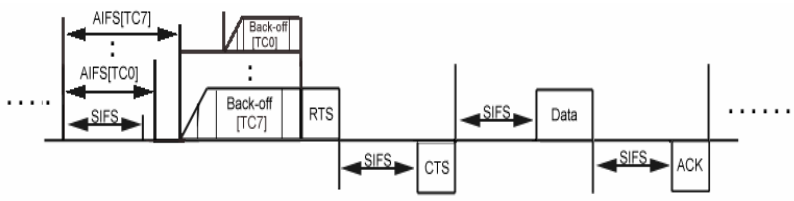

Figure 2: RTS/CTS of IEEE 802.11e EDCA

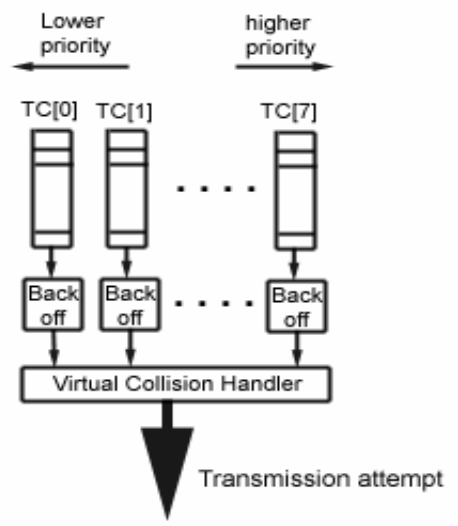

Figure 3: IEEE 802.11e MAC transmission queue

After experiencing any unsuccessful transmission, the new $c w$ is calculated as follows

$$
\left.n e w \_c w[\mathrm{TC}]>=((\text { oldcw }[\mathrm{TC}]-1) * \operatorname{Pf}[\mathrm{TC}])-1\right)
$$

where $P f[\mathrm{TC}]$ is the Persistence Factor that determines the degree in increase of the $c w$ in the event of collisions. Higher priority $\mathrm{TC}$ has smaller $P f[\mathrm{TC}]$ value.

\subsection{Traffic models}

Effective and reliable performance evaluation of the EDCA protocol requires appropriate traffic models that can accurately capture the properties of the real-world network traffic. The conventional Poisson model with which the inter-arrival times between packets are exponentially distributed has been widely used owing to its analytical simplicity [11, 12]. However, this model cannot capture bursty nature of network traffic, such as that generated by voice sources. The widely adopted model for voice traffic is the well-known bursty On-Off model where the talkspurt is modeled by ON stage and silence period is modeled by OFF stage. Both the ON and OFF periods follow an exponential distribution [5].

Extensive measurement studies on high-speed video traffic have reached to the conclusions that the VBR video traffic possesses self-similar properties. Self-similar traffic is characterized by long-range dependence where the pattern of packet generation appears similar when viewed over a wide range of time scales. Formally, a continuoustime process $X=\{X(t), t \in T\}$ is self-similar (with Hurst parameter $H)$ if it satisfies the condition: $X(t)=a^{-H} X(a t), \quad \forall t \in T, \quad \forall a>0, \quad$ and $\quad 0 \leq H<1$. Hurst parameter is used to characterize the degree of traffic self-similarity. Short-range dependent (SRD) processes have $H=0.5$ while Long-range dependent (LRD) processes have $0.5<H<1$.

\section{Simulation Scenarios and Configuration}

The well-known network simulator NS-2 with its wireless extensions developed by TKN [16] has been adopted to conduct our simulation experiments. This section will present the experiment scenarios and how to configure simulation parameters.

\subsection{Scenarios}

The simulation scenarios studied in this research was designed to investigate the performance of EDCA in hot spot areas of WLANs. As illustrated in Figure 5, the scenarios are composed of up to 20 mobile stations and an access point serving as a traffic sink. Each wireless station operates under the IEEE 802.11a standard at a data rate of 24Mbps [17]; the setting of physical layer (PHY) parameters is shown in Table 1. All stations are located within an Independent Basic Service Set (IBSS) such that every station is able to detect a transmission from others. 
Due to different types of services supported in WLANs, the simulation adopts heterogeneous traffic models to capture the characteristics of various wireless applications. Each station involves four types of traffic sources. The highest priority traffic (generated by voice sources) is modelled by the bursty ON/OFF process. In general, the talkspurt and silence durations of voice ON/OFF process are assumed to follow an exponential distribution with the mean value of $1 \mathrm{~s}$ and 1.35s [5], respectively. During the talkspurts period each source generates 80 byte-long packets, corresponding to a constant sending rate of 64 $\mathrm{kbit} / \mathrm{s}$. Considering there might be multiple voice source, we assume there are five voice sources at each station.

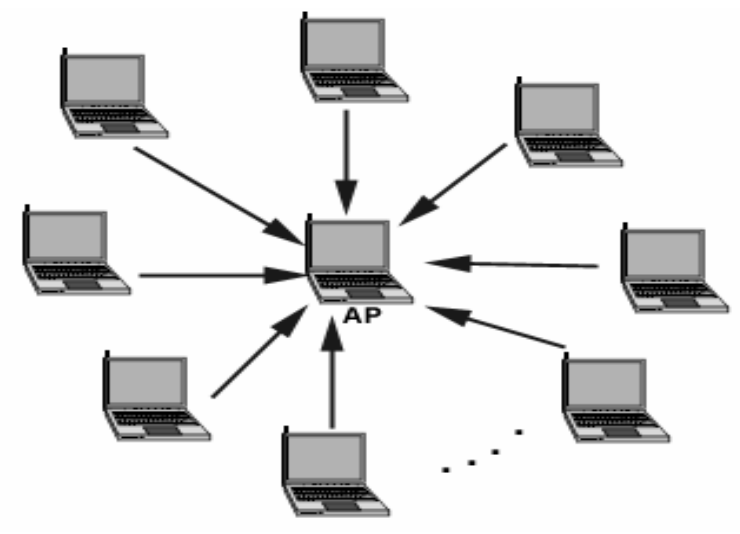

Figure 5: AP with mobile stations

\begin{tabular}{|l|l|}
\hline SlotTime & $9 \mu \mathrm{s}$ \\
\hline CCATime & $3 \mu \mathrm{s}$ \\
\hline RxTxTurnaroundTime & $2 \mu \mathrm{s}$ \\
\hline SIFSTime & $16 \mu \mathrm{s}$ \\
\hline PreambleLength & $96 \mathrm{bits}=16 \mu \mathrm{s}$ \\
\hline PLCPHeaderLength & $40 \mathrm{bits}$ \\
\hline PLCPDataRate & $6^{*} 10^{6} \mathrm{Mbps}$ \\
\hline
\end{tabular}

Table 1: NS-2 PHY parameters for IEEE 802.11a
Traffic generated by streaming applications such as real-time video and best-effort non-real-time video is modelled by self-similar processes. Following the measurement results on Star Wars video traffic [2, 4], video traffic rate is set to $360 \mathrm{kbit} / \mathrm{s}$ and Hurst parameter to 0.74 . Self-similar traffic can be generated by the superposition of many ON-OFF sources in which the ON and OFF periods follow a Pareto distribution (i.e., with high variability or infinite variance) [13]. In the simulation experiments, we used the superposition of five Paretodistributed ON-OFF flows with the average ON period of $10 \mathrm{~ms}$ and OFF period of $100 \mathrm{~ms}$ to generate self-similar traffic.

Besides, each station generates background data packets according to Poisson model (characterized by the independent exponentially-distributed inter-arrival times with sending rate equal to $160 \mathrm{Kbit} / \mathrm{s}$ and 200 bytes packet size; these parameters have been widely used in the previous network performance evaluation studies [11,12]. The priority of voice, real-time video, best-effort video, and background traffic follows a descending order. To support differentiated QoS of these traffic categories, EDCA protocol parameters including $c w_{\min }, c w_{\max }$, AIFS are selected for the four TCs based on the IEEE $802.11 \mathrm{e}$ standard and are listed in Table 2

\section{Performance Analysis and Evaluation}

The simulation work aims to investigate the prioritisation capability of the IEEE 802.11e EDCA MAC protocol, as well as the cost and overhead of prioritisation in terms of medium utilization, access delay and throughput. The average throughput is calculated as the amount of data that is actually delivered to the destination at each time unit. Many factors affect the throughput, including the efficiency of collision avoidance, medium utilization, latency and control overhead. The access delay is defined as the time elapsed between the arrival of a packet from the higher layer to the MAC layer and the successful transmission on wireless medium. We measure the mean and variance of the access delay to find out how well the EDCA accommodates real-time traffic especially

\begin{tabular}{|l|l|l|l|l|}
\hline Parameters & Voice & Real-Time Video & Best-Effort Video & Background \\
\hline$c w_{\min }$ & $\left(c w_{\min }+1\right) / 4-1=3$ & $\left(c w_{\min }+1\right) / 2-1=7$ & $c w_{\min }=15$ & $c w_{\min }=15$ \\
\hline$c w_{\max }$ & $\left(c w_{\min }+1\right) / 2-1=7$ & $c w_{\min }=15$ & $c w_{\max }=1023$ & $c w_{\max }=1023$ \\
\hline AIFS & 2 & 2 & 3 & 7 \\
\hline
\end{tabular}

Table 2: EDCA protocol parameters for different traffic categories 
the voice and video traffic. Medium utilization is referred to the percentage of time that is used for successful transmission.

\subsection{Throughput}

Figure 7 depicts the throughput of the different traffic categories versus the number of active stations in order to reveal the ability of the EDCA to support QoS differentiation. While the number of stations is small, we observe that the throughput gain of all traffic classes increases as the loads increase. By further increasing the number of active stations more than 7 , background traffic begins to decrease significantly due to its lowest priority. As a result, the background traffic cannot take the chance to use the wireless medium more than other higher priority traffic categories. However, the best-effort video traffic, which has the higher priority than background traffic, can carry on its load one station more. Moreover, it can be seen that the throughput of the real-time video streams keeps increasing until there are 9 active stations. Beyond this point the wireless medium cannot serve additional real-time streams and the throughput starts to decreases due to the large number of collisions caused by voice and real-time video traffic. Furthermore it can be observed that beyond 12 active stations the throughput of the voice streams cannot keep increasing and starts to drop down slightly. In addition, the throughput of the real-time video streams decreases more than that of the voice streams owing to the difference of their priorities. It is worth noting that the real-time streams can keep be served due to the fact that their contention windows are chosen from a relatively smaller interval range, so it can take the higher opportunities to capture the channel compared to besteffort video and background traffic.

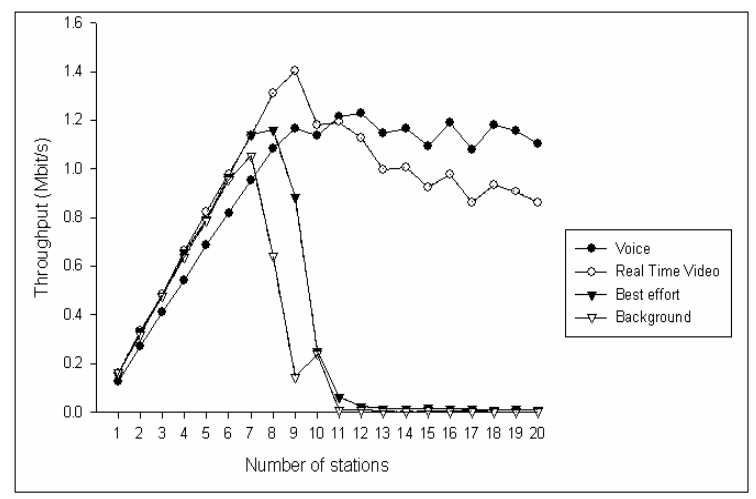

Figure 7: Throughput of the different traffic categories

\subsection{Access delay}

Figures 8 and 9 reveal the mean and variance of the access delay experienced by different traffic categories versus the number of active stations. These figures have shown that the background and best-effort traffic suffers from the high access delay and jitter because of their lower priorities. When the number of active stations is beyond 6 , the best effort and background traffic has the rare opportunity to capture the wireless medium so their delay is going to become infinity when the number of active stations is more than 7 .

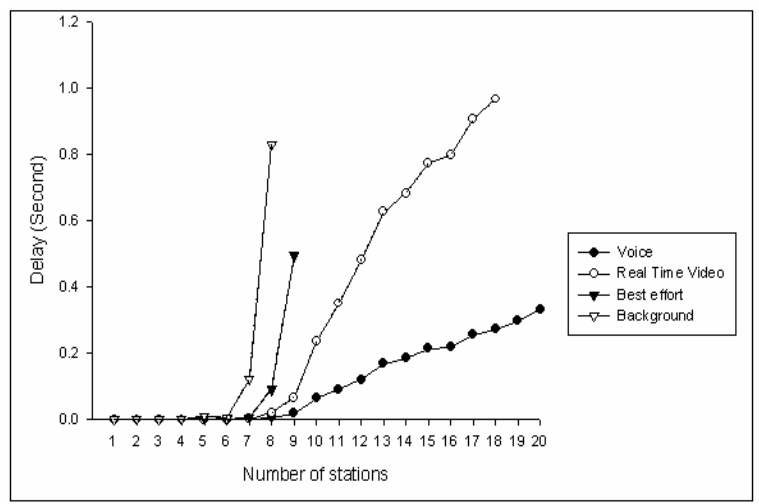

Figure 8: Average access delay of the different traffic categories

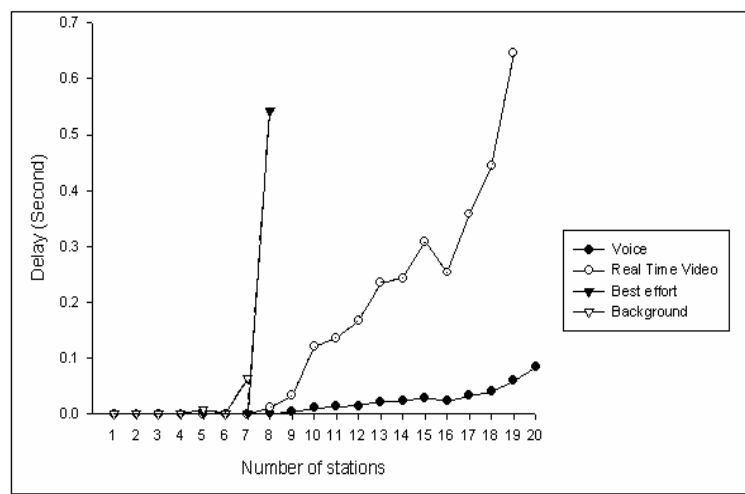

\section{Figure 9: Variance of the access delay of the different traffic categories}

With the increase in the number of active stations, the mean delay of voice and real-time video streams starts to increase when the network supports more than 7 active stations. This is because such traffic has to wait in the queue longer than before as the sequence of the increase in transmission collisions. The average delay and variance of real-time video traffic reaches to $350 \mathrm{~ms}$ and $120 \mathrm{~ms}$, respectively, and keeps rising up in a dramatic way when the number of active stations is beyond 10. It is also 
interesting to see that the delay experienced by the voice and real-time video is much different because the latter has to wait more time than the former until taking the opportunity to utilize the wireless medium.

\subsection{Medium utilization}

In addition to throughput and access delay, we further evaluate the performance of EDCA in terms of medium utilization. As shown in Figure 10, under all loads voice traffic always occupies the largest amount of the medium capacity among the four traffic categories. The medium utilization of voice traffic keeps rising as the load increases until there are 9 active stations in the network. After this point the trend of increase disappears and the utilization keeps around $16 \%$. It is also clear that real-time video traffic obtains better medium utilization than background and best-effort video traffic and keeps growing up until there are 9 active stations. After that it begins to lose some medium utilization because of giving the chance for the highest $\mathrm{TC}$ (voice traffic) to occupy the medium. Moreover, Figure 10 reveals the utilization of two lowest priority categories (background and best-effort traffic) decreases dramatically after there are 7 active stations. It is worth noting that the maximum total medium utilization of the WLAN is very low (around 40\%). This is because much capacity is wasted during the process of packet collisions and back-off. The increasing probability of packet collisions under heavy traffic can significantly degrade the medium utilization of the system.

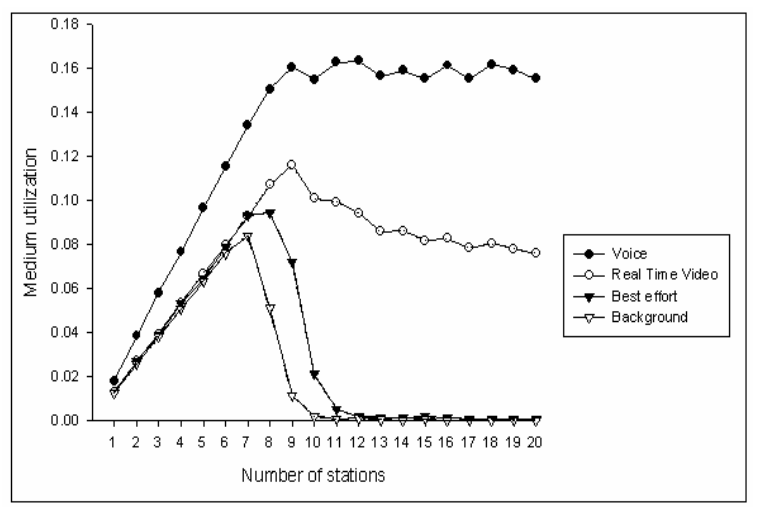

Figure 10: Medium utilization of the different traffic categories

\section{Conclusions}

The IEEE 802.11e EDCA mechanism was proposed for enhancing the traditional 802.11 DCF MAC protocols with QoS facility. Performance studies on the EDCA protocol have been widely conducted and reported in the literature. To the best of our knowledge, there has not work reported for the comprehensive performance evaluation and analysis of the IEEE 802.11e EDCA protocol in the presence of heterogeneous non-bursty Poisson, bursty ON/OFF, and self-similar traffic with wireless multimedia applications. To fill this gap, this study has used the well-known network simulator NS-2 to evaluate the performance of IEEE 802.11e MAC protocol in WLANs under heterogeneous traffic. Results obtained through simulation experiments have shown that this protocol can support differentiated throughput, access delay and medium utilization among various traffic categories. On the other hand the simulation experiments have showed that IEEE 802.11e EDCA suffering from the low medium utilization due to the overhead generated by transmission collisions and back-off processes. The next step in our work is to study the effect of hidden nodes on WLANs and multi-hop WLANs in the presence of heterogeneous traffic.

\section{Acknowledgements}

The research was supported in part by the UK EPSRC Grant EP/C525027/1.

\section{References}

[1] I. Aad and C. Castelluccia, Differentiation mechanisms for IEEE 802.11, Proc.20th Annual Joint Conference of the IEEE Computer and Communications Societies, IEEE (INFOCOM'2001), vol. 1, pp. 209-218, 2001.

[2] J. Beran, R. Sherman, M.S. Taqqu, and W. Willinger, Long-range dependence in variable-bit-rate video traffic, IEEE Trans. Communications, vol. 43, pp. 1566-1579, 1995.

[3] S. Choi, J. D. Prado, S. Shankar, and S. Mangold, IEEE 802.11e Contention-based channel access (EDCF) performance evaluation, Proc. IEEE Information and Communication Conference (ICC'2003), vol. 2, pp. 11511156, 2003.

[4] B.Chen, Z. Lucic, and L. Trajkovic, Simulation and wavelet analysis of packet traffic, http://www.ensc.sfu.ca/people/faculty/ljilja/cnl/pdf/bruce_ asi2002.pdf, 2005.

[5] R. Cox, Three new speech coder from the ITU cover a range of applications, IEEE Communication Magazine, vol. 35, no. 9, pp. 40-47, 1997.

[6] A.Grilo and M. Nunes, Performance evaluation of IEEE 802.11e Proc. 13th IEEE International Symposium, Personal, Indoor and Mobile Radio Communications (PIMRC'2002), vol. 1, pp. 511-517, 2002.

[7] M. Garrett and W. Willinger, Analysis, modelling and generation of self-similar VBR video traffic, Proc. Conference on Communications Architectures, Protocols and Applications, ACM Special Interest Group on Data Communication (SIGCOMM'1994), pp. 269-280, 1994.

[8] M. Gast, 802.11 wireless networks: the definitive guide, vol.1, O'Reilly, 2002.

[9] A.Lindgren, A. Almquist and O. Schelen, Evaluation of quality of service schemes for IEEE 802.11 wireless 
LANs, Proc. 26th Annual IEEE Conference on Local Computer Networks (LCN'2001), pp. 348 - 351, 2001.

[10] A.Lindgren, A. Almquist and O. Schelen, Quality of service schemes for IEEE 802.11 - a simulation study, Proc. 9th International Workshop on Quality of Service (IWQoS'2001), pp. 281-287, 2001.

[11] S. Mangold, S. Choi, G. Hiertz, O. Klein, and B. Walke, Analysis of IEEE $802.11 \mathrm{e}$ for QoS support in wireless LANs, IEEE Wireless Communications, vol. 10, no. 6, pp. 40-50, 2003.

[12] S. Mangold, S. Choi, Peter May, O. Klein,G. Hiertz, and L. Stibor, IEEE 802.11e wireless LAN for quality of service, Proc. European Wireless Conference, Italy, 2002.

[13] V. Paxson and S. Floyd, Wide-area traffic: the failure of poisson modeling, IEEE/ACM transaction on networking, vol. 3, no. 3, pp. 226-244, 1995.

[14] O. Tickoo, and B. Sikdar, On the impact of IEEE 802.11 MAC on traffic characteristics, IEEE Journal on Selected
Areas in Communications, vol. 21, no. 2, pp. 189-203, 2003.

[15] H. L. Truong and G. Vannuccini, Performance evaluation of the QoS enhanced IEEE 802.11e MAC layer, Proc. 57th IEEE Semi-annual, Vehicular Technology Conference (VTC'2003), vol.2, pp. 940-944, 2003.

[16] S. Wietholter and C. Hoene., Design and verification of an IEEE 802.11e EDCF simulation model in NS-2.26, technical report TKN-03-019, Telecommunication Networks Group, Technical University Berlin, November, 2003.

[17] IEEE WG 802.11, Part 11: Wireless LAN Medium Access Control (MAC) and Physical Layer (PHY) Specifications, ISO/IEC 8802-11:1999(E), IEEE Standards 802.11, 1999.

[18] IEEE WG 802.11, Part 11: Wireless LAN Medium Access Control (MAC) and Physical layer (PHY) specifications, Amendment 8: Medium Access Control (MAC) Quality of Service Enhancements, IEEE Standards 802.11e, 2005. 